\title{
Metachronous, colitis-associated rectal cancer that developed after sporadic adenocarcinoma in an adenoma in a patient with longstanding Crohn's disease: a case report
}

\author{
Hiroshi Takeyama, Tsunekazu Mizushima*, Kiyokazu Nakajima, Mamoru Uemura, Naotsugu Haraguchi, \\ Junichi Nishimura, Taishi Hata, Ichiro Takemasa, Hirofumi Yamamoto, Yuichiro Doki and Masaki Mori
}

\begin{abstract}
Background: Colorectal cancer associated with Crohn's disease (CD) is increasing in proportion to the number of patients with CD in Japan. There are two subtypes of colorectal cancer with CD: sporadic cancer and colitis-associated cancer. Early diagnosis of colitis-associated cancer is sometimes difficult; when colorectal cancer is found in patients with CD, both colitis-associated cancer and sporadic cancer should be kept in mind. Here, we describe a case of metachronous, colitis-associated rectal cancer that developed after the complete resection of an adenoma that became a sporadic adenocarcinoma in a patient with longstanding CD. To the best of our knowledge, this is the first report of colitis-associated cancer in a patient with CD after removal of a sporadic cancer.

Case presentation: We describe a 51-year old man with CD who had difficulty in defecation. A rectal polyp was detected and a transanal resection of the polyp was performed. A histopathological examination showed an adenoma with sporadic adenocarcinoma. After three years, a follow-up colonoscopy revealed a reddish, elevated lesion in the patient's rectum. A colonoscopic biopsy showed a signet ring cell carcinoma. We performed an abdominoperineal resection of the rectum and a bilateral pelvic lymph node dissection. A histopathological examination revealed a mucinous adenocarcinoma with signet ring cell carcinoma and lymph node metastasis. The patient received adjuvant chemotherapy with oral uracil $224 \mathrm{mg}$ combined with tegafur $100 \mathrm{mg}$ plus leucovorin. No signs of recurrence were noted at a follow-up 18 months after the third surgery and 60 months after the second surgery.
\end{abstract}

Keywords: Colitis-associated cancer, Crohn's disease, Sporadic cancer

\section{Background}

Colorectal carcinoma (CRC) is a serious complication of inflammatory bowel disease (IBD). CRC accounts for approximately $15 \%$ of all deaths associated with IBD [1]. CRC has been thoroughly studied in conjunction with ulcerative colitis (UC) [2], but CRC is not well understood in conjunction with Crohn's disease (CD). Several reports have described an increased incidence of CRC in CD [3-5]. However, relatively few studies have focused on CRCs in CD. No well-defined risk factors have been associated with the development of CRC in CD. Moreover, we lack clear recommendations for surveillance and surgical strategies for addressing CRC in CD [6]. CRC in patients with IBD can be classified into two subtypes, colitis-associated cancer and sporadic cancer, with different pathogenic and clinicopathological characteristics [6]. Few reports have precisely described each type of CRC in CD. To the best of our knowledge, no report has described metachronous sporadic and colitis-associated cancers in the same patient. This study is the first to report colitis-associated adenocarcinoma in a patient with $\mathrm{CD}$ after the removal of a sporadic adenocarcinoma.

\footnotetext{
* Correspondence: tmizushima@gesurg.med.osaka-u.ac.jp

Department of Surgery, Gastroenterological Surgery, Graduate School of

Medicine, Osaka University, 2-2 Yamada-Oka, Suita, Osaka 565-0871, Japan
} 


\section{Case presentation}

The patient was a 51-year old man with $\mathrm{CD}$. He was initially diagnosed with $\mathrm{CD}$ at 23 years old. He had undergone ileocecal resection, owing to a stricture of the terminal ileum, at 36 years old, and the residual small intestine was $230 \mathrm{~cm}$ long. After the initial operation, he was prescribed 5-aminosalicylic acid and home parenteral nutrition. He did not accept a recommended colonoscopy examination, owing to anal pain.

In August 2008, the patient complained of difficulty in defecation and consulted a physician at our hospital. A colonoscopy examination showed a pedunculated rectal polyp, $4 \mathrm{~cm}$ in diameter (Figure 1) and an anal stricture due to proctitis. Histological examination of the biopsy from the lesion showed an adenoma; no dysplasia was detected around the polyp. A transanal resection of the polyp was performed for treatment and final diagnosis (Figure 2). The pathological findings revealed that the polyp was an adenoma with well-differentiated adenocarcinoma without lymphatic or vascular invasion with negative margin of cut end, and that no dysplasia or inflammation was present around the polyp; thus, the polyp was diagnosed as a sporadic adenocarcinoma in an adenoma, rather than a colitis-associated adenocarcinoma (Figure 3). Consistent with the results of the biopsy, an endoscopic exploration showed no dysplasia in the rectal mucosa, and immunohistochemistry showed no p53-staining of the crypt base. These findings were not consistent with colitis-associated CRC (Figure 4). In July 2010, follow-up colonoscopy showed a longitudinal ulcer scar from the descending colon to the ascending colon and stenosis of the ileocolic anastomosis. We did not find any apparent lesion in the rectum.

Three years after the second surgery, in August 2011, colonoscopy examination revealed a reddish, elevated

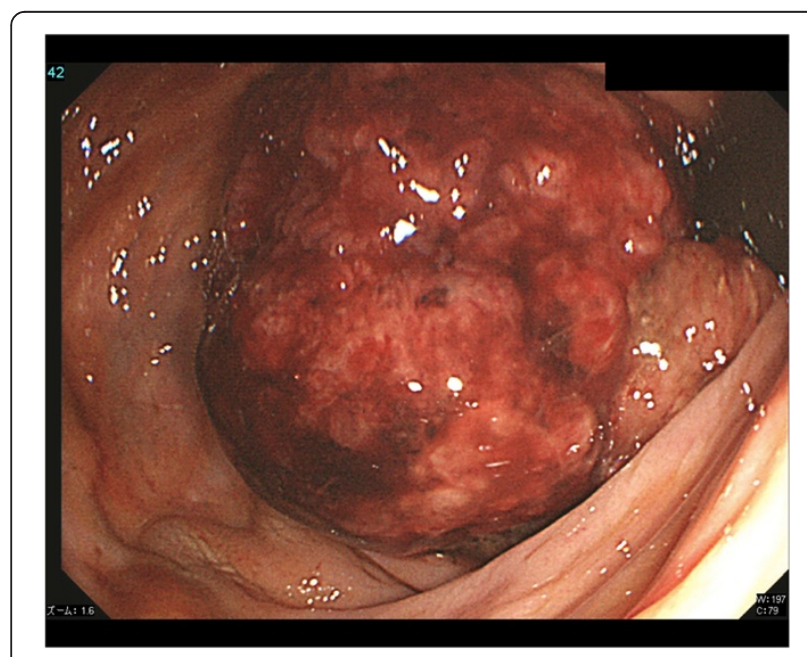

Figure 1 Colonoscopic image shows a rectal polyp, $4 \mathrm{~cm}$ in diameter.

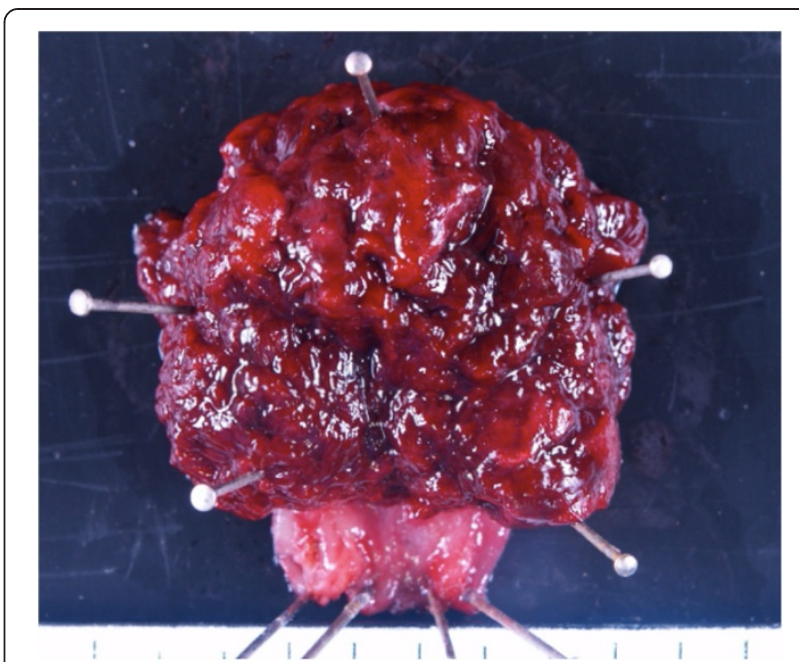

Figure 2 Image of the surgical specimen shows the excised rectal polyp, $4 \mathrm{~cm}$ in diameter.
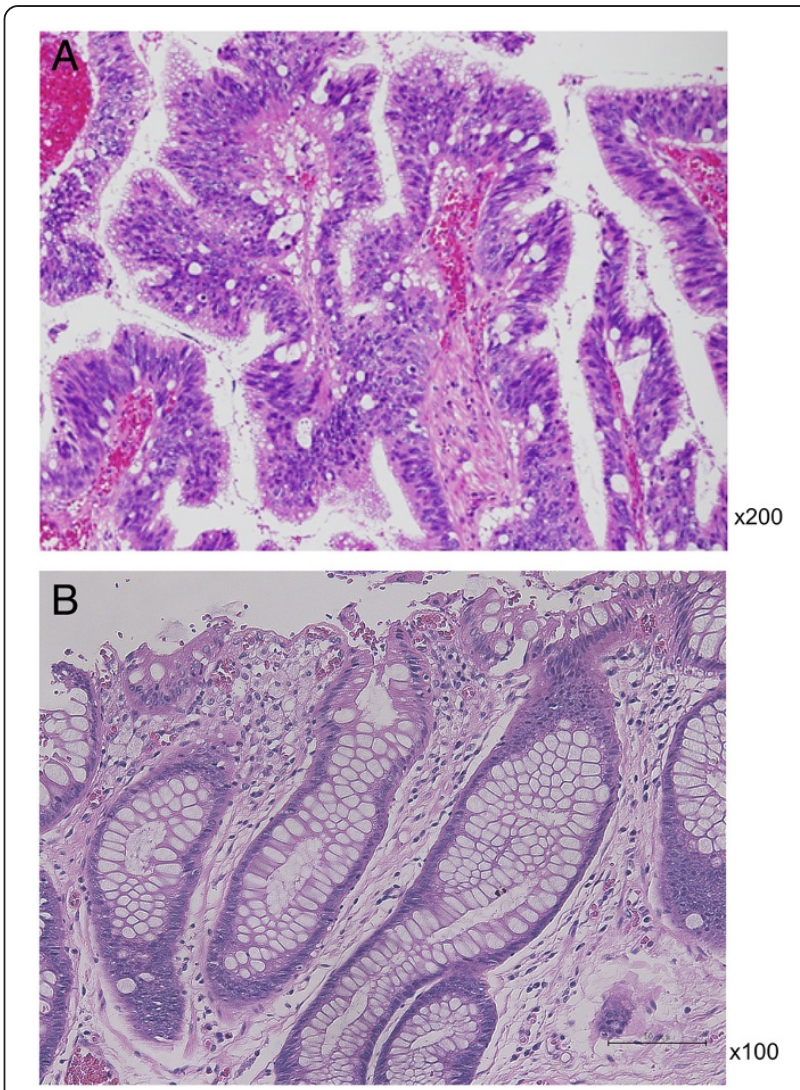

Figure 3 Histopathological images. (A) The polyp was diagnosed as sporadic adenocarcinoma in an adenoma, rather than colitis-associated adenocarcinoma ( $\times 200, H$ \& E). (B) Section of tissue that bordered the polyp; there is no dysplasia or inflammation ( $\times 100, H$ \& E stain). 


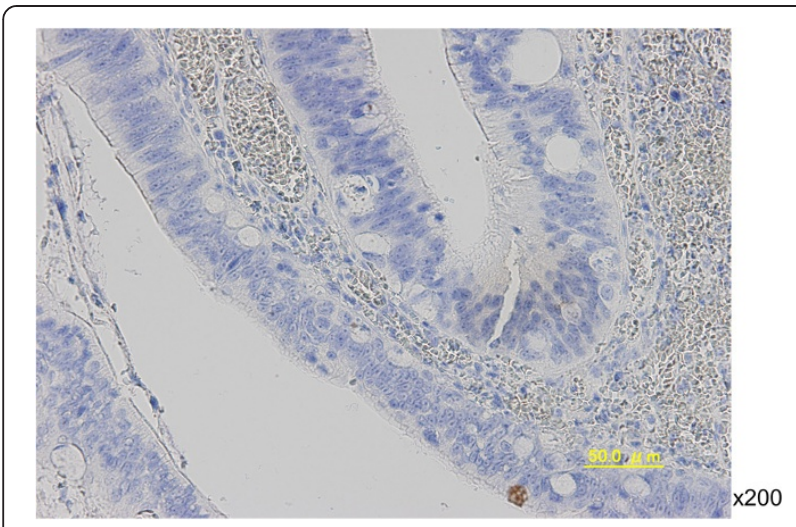

Figure 4 Immunohistochemical staining for p53 in rectal mucosa tissue shows no dysplasia in the crypt base. These findings were not consistent with a colitis-associated colorectal cancer $(\times 200)$.

lesion in the rectum (Figure 5). This lesion was located at a different site from the initial lesion. A colonoscopic biopsy revealed a signet ring cell carcinoma. We performed an abdominoperineal resection of the rectum and a bilateral pelvic lymph node dissection (Figure 6). Postoperative histological examination showed a mucinous adenocarcinoma with signet ring cell carcinoma and lymph node metastasis (Figure 7). Dysplasia was detected in the rectal mucosa, and the crypt base was immunohistochemically stained with $\mathrm{p} 53$. These findings were consistent with colitis-associated CRC (Figure 8).

Postoperatively, the patient received adjuvant chemotherapy with $224 \mathrm{mg}$ oral uracil combined with100 $\mathrm{mg}$ tegafur plus leucovorin for 6 months. The patient has continued with regular follow-ups over the last 18 months after the third surgery and over the last 60 months after

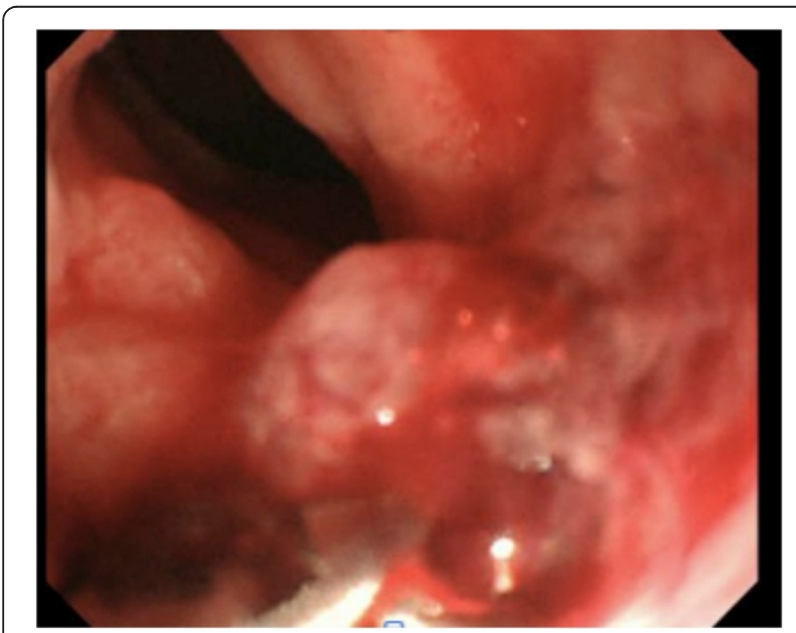

Figure 5 Colonoscopic image shows a reddish, elevated lesion in the rectum. This was located in a site different site from the initial lesion. the second surgery. He has also undergone regular examinations. A regular colonoscopy examination was performed every 6 months, and no relapse of colonic inflammation or dysplasia was detected.

\section{Discussion}

CRC accounts for approximately $15 \%$ of all deaths in patients with IBD $[6,7]$. The risk of CRC for patients with IBD increases by $0.5 \%$ to $1 \%$ yearly from 8 to 10 years after the IBD diagnosis. A meta-analysis of published studies that focused on the CRC risk in UC showed that the risk of CRC for patients with colitis was $2 \%$ at 10 years, $8 \%$ at 20 years, and $18 \%$ after 30 years of colitis [8]. Previously, CD was not considered a risk factor for CRC; however, recent studies have shown that the CRC risk in patients with colonic $\mathrm{CD}$ is similar to that of patients with UC $[7,9]$.

In patients with IBD, CRC can take the form of sporadic cancer or colitis-associated cancer. In patients with UC who develop colitis-associated cancer, total proctocolectomy is the standard treatment; however, when sporadic cancer occurs, total proctocolectomy is not always necessary. In patients with $\mathrm{CD}$, an indication for total proctocolectomy has been controversial. However, it was also reported that, when a diagnosis of colitis-associated cancer is known in advance of surgery, a total proctocolectomy should be seriously considered, to eliminate the risk of metachronous disease. In $44 \%$ of these patients, dysplasia occurred at a site remote from the site of the cancer [10]. However, this patient had already undergone ileocecal resection and the residual small intestine was $230 \mathrm{~cm}$ long, resulting in intestinal failure syndrome requiring home parental nutrition. For these reasons, we chose abdominoperineal resection with colostomy, not total proctocolectomy with ileostomy, in order to preserve the colon and maintain absorption of water and electrolytes. After the last surgery, we conducted intensive cancer surveillance and neither a recurrence nor another lesion has been detected at this time.

However, the site of inflammation should be resected, owing to the risk of dysplasia. When the colon and rectum are preserved in surgery, a scheduled, annual surveillance with biopsies is strongly recommended [10]. It is very important, when planning therapy and surveillance after surgery, to perform a differential diagnosis to distinguish between colitis-associated CRC and sporadic CRC. However, it is often difficult to achieve this differential diagnosis in patients with IBD, particularly before surgery $[11,12]$.

The American Gastroenterological Association and the British Society for Gastroenterology surveillance guidelines have recommended beginning surveillance after 8 to 10 years of disease in cases of $\mathrm{CD}$ or extensive $\mathrm{UC}$, and after 15 to 20 years of disease in cases of left- 


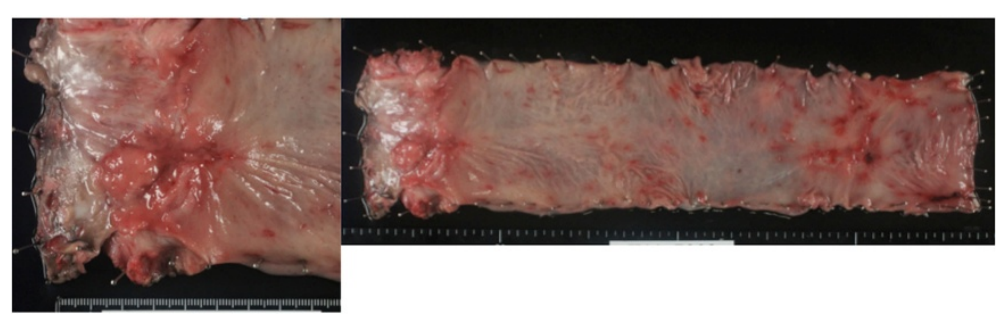

Figure 6 Image of the surgical specimen shows a reddish and elevated lesion, $3 \mathrm{~cm}$ in diameter.

sided UC [13-15]. However, the evidence on which this is based on is poor. It was reported that cancer has occurred in a substantial number of patients with IBD ( $17 \%$ to $28 \%$ ) before the recommended time intervals for surveillance [16].

Clinically, endoscopic examination is the most useful screening method. However, even with endoscopic examination, it is often difficult to detect dysplastic changes. Colitis-associated dysplasia may show subtle macroscopic features that mimic a broad variety of lesions, ranging from inflammation to carcinoma. Previous reports have indicated that dysplastic lesions were not visible upon endoscopy in $50 \%$ to $80 \%$ of cases with colitis-associated cancers [17]. Conversely, sporadic cancer mainly develops from polyps that are on the pathway to becoming adenoma-carcinomas, and a polyp is easily detectable, even in a normal endoscopy examination. In contrast, the gross appearance of colitis-associated neoplasms varies from case to case, as the neoplasm develops from dysplasia to become a carcinoma. Dysplastic lesions may be irregularly delineated, plaque-like, irregularly elevated, or verrucous structures; therefore, they are difficult to identify with normal endoscopy [18].

This case should make surgeons and endoscopists aware of the possibility that patients with $C D$ may

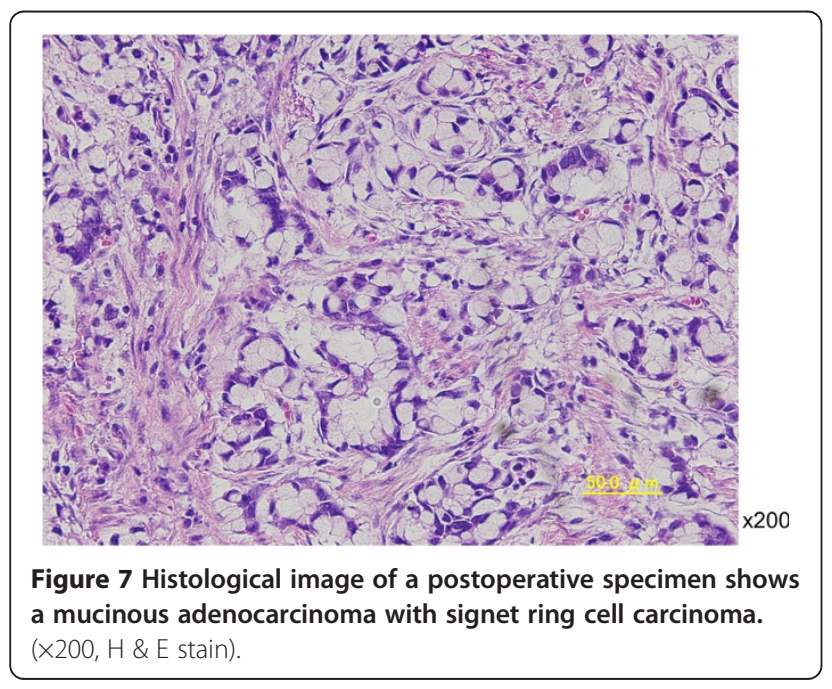

develop either colitis-associated cancer or sporadic cancer. This report should alert surgeons and endoscopists to look for colitis-associated cancers that are in a precancerous or early stage. In this case study, although we performed annual colonoscopic surveillance, we did not detect the second lesion, the colitis-associated cancer, until it reached an advanced cancerous stage. Early detection is highly important for avoiding surgeries, including colectomy or proctectomy. However, it is often difficult to detect colitis-associated cancers in dysplastic, precancerous, or early stages. We need further investigation to discover novel biomarkers that make it possible to detect cancer in blood samples or biopsies.

\section{Conclusions}

To the best of our knowledge, this is the first case report of a metachronous colitis-associated rectal cancer that arose in a patient with $\mathrm{CD}$ after removal of a sporadic cancer. This case emphasized the importance for clinicians to bear in mind the possibilities of both sporadic cancer and colitis-associated cancer when following-up patients with $\mathrm{CD}$. It is also important to consider surgical therapy and surveillance after surgery, and to perform a differential diagnosis to distinguish between colitis-associated cancer and sporadic cancer. CRC

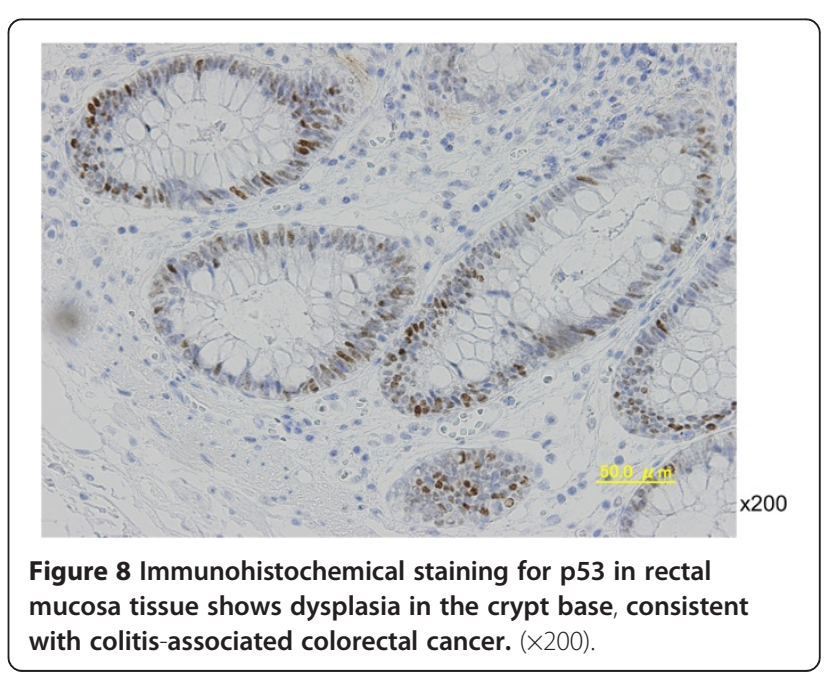


associated with $\mathrm{CD}$ remains a rare occurrence, and there is no defined surveillance strategy. However, patients with longstanding CD should receive cancer surveillance as frequently as do patients with UC.

\section{Consent}

Written informed consent was obtained from the patient for publication of this case report and accompanying images. A copy of the written consent is available for review by the editor-in-chief of this journal.

\section{Abbreviations}

CD: Crohn's disease; CRC: Colorectal carcinoma; H \& E: Hematoxylin and eosin; IBD: Inflammatory bowel disease; UC: Ulcerative colitis.

\section{Competing interests}

There are no conflicts of interest to disclose for any author.

\section{Authors' contributions}

$\mathrm{HT}$ wrote the main manuscript, and TM and KN performed the operation, revised the manuscript for important intellectual content, and gave the fina approval for the version to be submitted for publication. All authors read and approve the final manuscript.

Received: 30 April 2013 Accepted: 9 November 2013

Published: 19 November 2013

\section{References}

1. Munkholm P: Review article: the incidence and prevalence of colorectal cancer in inflammatory bowel disease. Aliment Pharmacol Ther 2003, 18(2):1-5.

2. Maratka Z, Nedbal J, Kocianova J, Havelka J, Kudrmann J, Hendl J: Incidence of colorectal cancer in proctocolitis: a retrospective study of 959 cases over 40 years. Gut 1985, 26:43-49.

3. Averboukh F, Ziv Y, Kariv Y, Zmora O, Dotan I, Klausner JM, Rabau M, Tulchinsky $\mathrm{H}$ : Colorectal carcinoma in inflammatory bowel disease: a comparison between Crohn's and ulcerative colitis. Colorectal Dis 2011 13:1230-1235.

4. Mpofu C, Watson AJ, Rhodes JM: Strategies for detecting colon cancer and/or dysplasia in patients with inflammatory bowel disease. Cochrane Database Syst Rev 2004, 2:CD000279.

5. Ruffolo C, Scarpa M, Polese L, D'Amico FE, Boetto R, Pozza A, D'Inca R, Checchin D, Sturniolo GC, Bassi N, Angriman I: Clinical presentation and diagnosis of intestinal adenocarcinoma in Crohn's disease: analysis of clinical predictors and of the life-time risk. J Gastrointest Surg 2010, 14:1746-1751.

6. Itzkowitz SH, Harpaz N: Diagnosis and management of dysplasia in patients with inflammatory bowel diseases. Gastroenterology 2004, 126:1634-1648.

7. Vagefi PA, Longo WE: Colorectal cancer in patients with inflammatory bowel disease. Clin Colorectal Cancer 2005, 4:313-319.

8. Eaden JA, Abrams KR, Mayberry JF: The risk of colorectal cancer in ulcerative colitis: a meta-analysis. Gut 2001, 48:526-535.

9. Bernstein $C N$, Blanchard JF, Kliewer E, Wajda A: Cancer risk in patients with inflammatory bowel disease: a population-based study. Cancer 2001. 91:854-862.

10. Kiran RP, Nisar PJ, Goldblum JR, Fazio WW, Remzi FH, Shen B, Lavery IC: Dysplasia associated with Crohn's colitis: segmental colectomy or more extended resection? Ann Surg 2012, 256:221-226.

11. Nagasako K, lizuka B, Ishii F, Miyazaki J, Fujimori T: Colonoscopic diagnosis of dysplasia and early cancer in longstanding colitis. J Gastroenterol 1995, 30(8):36-39.

12. Riddell RH, Goldman $H$, Ransohoff DF, Appelman HD, Fenoglio CM, Haggitt RC, Ahren C, Correa P, Hamilton SR, Morson BC, Sommers SC, Yardley $\mathrm{JH}$ : Dysplasia in inflammatory bowel disease: standardized classification with provisional clinical applications. Hum Pathol 1983, 14:931-968.
13. Eaden JA, Mayberry JF: Guidelines for screening and surveillance of asymptomatic colorectal cancer in patients with inflammatory bowel disease. Gut 2002, 51(5):V10-V12.

14. Kornbluth A, Sachar DB: Ulcerative colitis practice guidelines in adults: American College of Gastroenterology, Practice Parameters Committee. Am J Gastroenterol 2010, 105:501-523. quiz 24

15. Winawer $S$, Fletcher R, Rex D, Bond J, Burt R, Ferrucci J, Ganiats T, Levin T, Woolf S, Johnson D, Kirk L, Litin S, Simmang C: Colorectal cancer screening and surveillance: clinical guidelines and rationale-update based on new evidence. Gastroenterology 2003, 124:544-560.

16. Lutgens MW, Vleggaar FP, Schipper ME, Stokkers PC, van der Woude CJ, Hommes DW, der Jong DJ, Dijkstra G, van Bodegraven AA, Oldenburg B, Samsom M: High frequency of early colorectal cancer in inflammatory bowel disease. Gut 2008, 57:1246-1251

17. Bernstein CN, Shanahan F, Weinstein WM: Are we telling patients the truth about surveillance colonoscopy in ulcerative colitis? Lancet 1994, 343:71-74.

18. Neumann H, Vieth M, Langner C, Neurath MF, Mudter J: Cancer risk in IBD: how to diagnose and how to manage DALM and ALM. World J Gastroenterol 2011, 17:3184-3191.

doi:10.1186/1477-7819-11-295

Cite this article as: Takeyama et al:: Metachronous, colitis-associated rectal cancer that developed after sporadic adenocarcinoma in an adenoma in a patient with longstanding Crohn's disease: a case report. World Journal of Surgical Oncology 2013 11:295.

\section{Submit your next manuscript to BioMed Central and take full advantage of:}

- Convenient online submission

- Thorough peer review

- No space constraints or color figure charges

- Immediate publication on acceptance

- Inclusion in PubMed, CAS, Scopus and Google Scholar

- Research which is freely available for redistribution 